\title{
Effect of Lycopene on Bisphenol A-induced Immuno- logical Disturbances in Albino Male Rats
}

\author{
Heba MA. Abdelrazek', Dalia A. Eltamany ${ }^{2}$, Mohammed TA. Soliman³, \\ Sahar M. Greish ${ }^{*}$, Hala M. Ebaid ${ }^{5}$
}

${ }^{1}$ Department of Physiology, Faculty of Veterinary Medicine, Suez Canal University, Ismailia, Egypt. ${ }^{2} \mathrm{Nu}$ trition and Food Science, Home Economic Department, Faculty of Education, Suez Canal University, Ismailia, Egypt. ${ }^{3}$ College of Applied Medical Sciences, Department of Medical Laboratory Sciences, University of Bisha, Kingdom Saudi Arabia. ${ }^{4}$ Department of Physiology, Faculty of Medicine, Suez Canal University, Ismailia, Egypt. ${ }^{5}$ Department of Zoology, Faculty of Science, Suez Canal University, Ismailia, Egypt

\begin{abstract}
Background: Bisphenol A (BPA) is a widely used and environmentally spread plasticizer. BPA has various toxic effects, including immunological alterations. Aim: Current work aimed to investigate the effect of BPA and protection with lycopene (LYC) on immunological parameters. Material and Methods: Twenty-four male albino were divided into 4 equal groups. Control group received corn oil, LYC group was given LYC $5 \mathrm{mg} / \mathrm{kg}$, BPA group was given BPA at $5 \mathrm{mg} / \mathrm{kg}$. Last group was given combination of BPA and LYC the same previous doses. Treatments were given daily via oral gavage for 4 weeks. Weight gain, food conversion ratio (FCR), hematological parameters and lymphocytes proliferation assay were determined. Serum total antioxidant capacity (TAC), malondialdehyde (MDA), interleukin-1 $\beta$ (IL-1 $\beta$ ), Interleukin-12 (IL-12) and lymphocytes comet assay were evaluated. Spleen and thymus histopathological assessment was done. Results: BPA significantly $(P<0.05)$ increased $F C R$ while decreased lymphocytes proliferation than control group however, hematological parameters didn't alter. Serum TAC was significantly $(P<0.05)$ reduced in BPA group than control group while MDA, IL-1 $\beta$ and IL-12 were significantly $(p<0.05)$ increased than control group. Lymphocyte showed significant increase in comet tail and \% of damage. Thymus and spleen revealed mild lymphoid depletion. Conclusion: BPA induced immunological perturbations that favors pro-inflammation and autoimmunity. LYC administration ameliorated oxidative stress and lipid peroxidation induced immunological disorders.
\end{abstract}

Keywords: Antioxidant, bisphenol A, cytokines, Hematology, lycopene

\section{Introduction}

Bisphenol A (BPA) has chemical formula; 4, 4'-dihydroxy-2, 2- diphenyl propane. It is widely distributed organic compound in polycarbonate plastics and resins of epoxy. The polycarbonate plastics are widely used in manufacture of packaging food and drink cans as well as water and infant bottles and several household items ${ }^{(1)}$. This wide range of exposure provides 
continuous exposure to human as well as animals including wild animals to BPA thus BPA have been found in the general population ${ }^{(2)}$. Immune system is very important for human and animal survival. Immune system involves many types of organs, cells and proteins that spread all over the body tissues. It can attack and clear faulty or dead cells as well as infectious agents (3). Immunosuppression leads to shortening of creatures' life span via increasing their liability to infectious agents and down regulates immune response to non-self-antigens ${ }^{(4)}$. The BPA can be categorized as an endocrine disruptor as it binds to estrogen receptors. Endocrine disruptors make shift in immunological response via altering the two types of $\mathrm{T}$ - lymphocytes ( $T$ helper 1 and $T$ helper 2) (5). Moreover, there is common concept that BPA exert its various toxic effects, including immunological alterations, via oxidative stress induction and generation of reactive oxygen species (ROS) $(6,7)$. Therefore, it is important to comprehend, the influence of BPA on the immune system. In this view, using laboratory animal models could enable us to understand the clues of such effect ${ }^{(1)}$. Great interests have been revolted in utilization of natural products purified from plants against immunological disorders ${ }^{(8)}$. Lycopene (LYC) present in tomatoes and other foods. It is lipophilic carotenoid that gives red color to the tomato ${ }^{(9)}$. It has an antioxidant and free radical eliminating effect ${ }^{(10)}$. Supplementation of LYC had been investigated for its preventive effect to the harmful tissues oxidative injury resulted from several environmental toxicants $(11,12)$. The aim of this study is to clarify the potential effect of BPA on hematological parameters and im- munological response. Also, investigating the ameliorative effect of LYC as natural antioxidant on BPA immunological alterations.

\section{Materials and Methods}

Animals and experiment design

Twenty-four male albino rats weighing 100-105 g, were obtained from Organization for Biological Products and Vaccines, Helwan, Egypt. They were kept 1 week for accommodation before the onset of the experiment. Animals were kept in ventilated room under room temperature $25 \pm 2{ }^{\circ} \mathrm{C}$, natural light/dark rhythm and humidity $47 \% \pm$ 2.0 Rats received ad libitum drinking water and diet. Rats were randomly distributed to four groups; six rats each. Group I: control group, received corn oil control. Group II: LYC group, received LYC (NOW FOODS Co., USA) 5 $\mathrm{mg} / \mathrm{kg}$ via oral gavage daily. Group III: BPA group, received BPA (Sigma, Aldrich (o., USA) at $5 \mathrm{mg} / \mathrm{kg}$ via oral gavage daily. Group IV: BPA and LYC group, received combination of BPA and LYC at the same doses of groups II and III, via oral gavage daily. All treatments were given for a duration of 4 weeks.

Body weight and feed conversion ratio (FCR)

Experimental rats were weighed at day 1 of the experiment then weighed at the end of experimental period. Food intake was recorded during the experimental period. FCR was calculated as follow: $F C R=$ (Feed consumption (g)/rat/4 weeks)/ (body weight gain (g)/rat/4 weeks).

Blood and tissue sampling:

At the end of the experiment, the rats were anesthetized, and blood samples 
were collected from retro-orbital venous plexus of the eye into Ethylene diamine tetra acetic acid (EDTA) tubes, lithium heparinized tubes and plain tubes. The later tubes left for clotting, kept in refrigerator then sera were separated, collected and stored at $30^{\circ} \mathrm{C}$. Rats were sacrificed then spleen and thymus were excised and weighed.

\section{Hematology}

Blood samples in EDTA tubes were subjected to manual red blood cells (RBCS) count, packed cell volume (PCV), total leukocytes count (TLC) and differential leukocyte count (DLC). Also mean corpuscular hemoglobin $(\mathrm{MCH})$, mean corpuscular hemoglobin concentration $(\mathrm{MCHC})$ and mean corpuscular volume (MCV). All procedures were performed according to Chand(13).

Lymphocyte proliferation assay

Lithium heparinized blood samples were transferred immediately to the laboratory. The buffy coat was separated and washed with RPMI-1640 medium (Sigma-Aldrich, Egypt). The obtained lymphocytes were suspended in $1 \mathrm{ml} \mathrm{RPMI-1640}$ medium supplemented with $10 \%$ of fetal calf serum (Sigma-Aldrich, Egypt) according to Boyum (14) and Burrells and Wells (15). The viable lymphocytes per ml RPMI medium was estimated. Transformation assay of viable lymphocytes was performed using MTT staining step which assayed calorimetrically at $490 \mathrm{~nm}$ optical density ${ }^{(16)}$.

Lipid peroxidation and total antioxidant capacity (TAC)

Malondialdehyde (MDA) was determined in sera according to Mihara and Uchiyama (17). The TAC was estimated in sera according to the manufacturer's protocol (Labor Diagnostika Nord $\mathrm{GmbH} \&$ Co. KG Co., Germany).

\section{Cytokines assay}

Interleukin -1 $\beta$ (IL-1 $\beta$ ) and Interleukin12 (IL-12) were assayed using ELISA commercial kits (IBL Co., Japan). The procedures were followed according to manufacturer's protocol.

Histopathological examination:

Thymus and spleen were immersed in $10 \%$ Formalin buffer, paraffinized in blocks and cut into 5 - $\mu$ m-thickness sections. These sections were stained with hematoxylin and eosin (H\&E) according to Drury and Wallington (18) and examined under light microscope.

\section{Comet assay}

The procedures of comet assay used to test effect of BPA and co- treatment with LYC on DNA damage. Lymphocytes were separated by Ficoll and subjected to the comet procedures described by ${ }^{(19)}$. The whole steps were performed in dim light to minimize DNA damage artifacts. Analysis was done at a magnification of $100 \times$ via a light microscope. Minimum 100 cells were screened per each slide ${ }^{(16)}$.

\section{Statistical Analyses}

Statistical analyses were performed by One-way analysis of variance (ANOVA) followed by Duncan's multiple comparison tests (SPSS v. 16.0, SPSS Inc., IL, USA). All values were represented as mean \pm standard errors. A probability level of $p<0.05$ denoted significance.

\section{Results}

Body weight and feed conversion ratio (FCR) 
The body weight gain was not significantly altered among tested groups after 4 weeks. However, FCR was significantly $(p<0.05)$ increased in BPA group than control and other groups (Fig. 1)

\section{Hematological parameters}

RBCs number, PCV, Hb values, $\mathrm{MCH}$, $M C H C$ and MCV were non-significantly altered among all groups. Also, TLC and DLC, showed non-significant alterations among study groups (Table 1).

Lymphocyte proliferation assay

Mitogen proliferation assay of separated lymphocytes was significantly $(P<0.05)$ declined in BPA treated group than control and other groups. The BPA and LYC co-administrated group exhibited a significant $(P<0.05)$ improvement in lymphocytes transformation than BPA group (Table 2).

Lipid peroxidation and total antioxidant capacity (TAC)

The level of MDA was significantly $(p<0.05)$ elevated in BPA intoxicated rats than control while TAC was significantly $(p<0.05)$ declined in such group than control. Giving LYC with BPA to experimental rats significantly $(p<0.05)$ ameliorated the BPA adverse effects where MDA reduced, and TAC promoted than BPA group (Table 2).

Cytokines assay

The levels of both IL-1 $\beta$ and IL-12 were significantly $(P<0.05)$ elevated in BPA treated groups. Meanwhile the co-administration of LYC and BPA caused significant $(P<0.05)$ reduction in the level of IL-12 than BPA group to a level comparable to control (Table 2).

Histopathological examination:

Spleen of Control and LYC groups showed normal microscopic architecture of both white and red pulp with normal well-developed lymphoid follicles, blood sinusoids and reticuloendothelial cells. The BPA group showed marked multifocal depletion of lymphoid follicles along with mild to moderated congestion and perivascular edema. Spleen of LYC+BPA co-treated group, had a mild focal lymphocytic depletion (Fig. 2). Thymus of Control and LYC groups showed normal cortex and
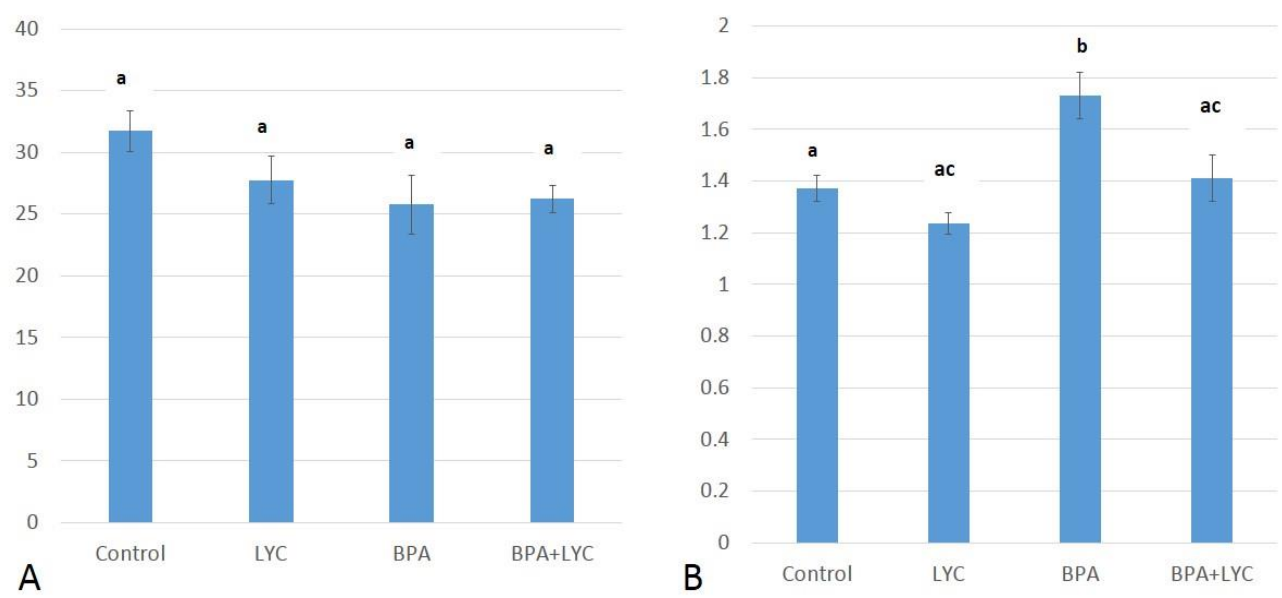

Figure 1: Effect of BPA and LYC on body weight gain (A) and FCR (B). Different superscripts between bars are considered significant at $p<0.05$. 
Table 1: Effect of BPA and LYC on hematological parameters.

\begin{tabular}{l|cccc}
\hline & Control & LYC & BPA & BPA+LYC \\
\hline $\mathrm{HB}(\mathrm{g} / \mathrm{dl})$ & $13.33 \pm 0.80$ & $14.53^{ \pm 0.95}$ & $13.6 \pm 0.61$ & $14.67 \pm 0.61$ \\
\hline $\mathrm{PVC}(\%)$ & $46.17 \pm 1.73$ & $46.27 \pm 5.71$ & $38.4 \pm 0.40$ & $44.70 \pm 3.55$ \\
\hline $\mathrm{RBCs}\left(10^{6} / \mu \mathrm{L}\right)$ & $4.82 \pm 0.08$ & $4.98 \pm 0.17$ & $4.47 \pm 0.25$ & $4.84 \pm 0.42$ \\
\hline $\mathrm{MCH}(\mathrm{pg})$ & $28.32 \pm 1.31$ & $30.53^{ \pm 1.30}$ & $33.08 \pm 1.85$ & $31.76 \pm 1.02$ \\
\hline $\mathrm{MCV}(\mathrm{fl})$ & $95.73 \pm 5.20$ & $92.47 \pm 4.21$ & $86.53 \pm 4.58$ & $92.41 \pm 3.95$ \\
\hline $\mathrm{MCHC}(\%)$ & $29.05 \pm 1.20$ & $32.07 \pm 0.98$ & $35.4 \pm 1.02$ & $33.00 \pm 2.01$ \\
\hline $\mathrm{TLC}\left(10^{3} / \mu \mathrm{L}\right)$ & $8.66 \pm 0.88$ & $11.10 \pm 1.89$ & $10.13 \pm 0.93$ & $12.60 \pm 1.94$ \\
\hline Neutrophils $\left(10^{3} / \mu \mathrm{L}\right)$ & $2.56 \pm 0.60$ & $3.30 \pm 0.48$ & $2.70 \pm 0.49$ & $4.95 \pm 1.40$ \\
\hline Lymphocytes $\left(10^{3} / \mu \mathrm{L}\right)$ & $5.53 \pm 1.37$ & $7.16 \pm 1.39$ & $6.84 \pm 0.43$ & $6.61 \pm 0.93$ \\
\hline Monocytes $\left(10^{3} / \mu \mathrm{L}\right)$ & $0.41 \pm 0.03$ & $0.49 \pm 0.06$ & $0.40 \pm 0.03$ & $0.58 \pm 0.07$ \\
\hline Eosinophil $\left(10^{3} / \mu \mathrm{L}\right)$ & $0.15 \pm 0.06$ & $0.14 \pm 0.03$ & $0.13 \pm 0.04$ & $0.29 \pm 0.10$ \\
\hline Basophils $\left(10^{3} / \mu \mathrm{L}\right)$ & $0.00 \pm 0.00$ & $0.00 \pm 0.00$ & $0.03 \pm 0.03$ & $0.03 \pm 0.03$ \\
\hline
\end{tabular}

Table 2: Effect of BPA and LYC on lymphocytes transformation, cytokines (IL-1 $\beta$ and IL-12), TAC and MDA

\begin{tabular}{l|cccc}
\hline & Control & LYC & BPA & BPA+LYC \\
\hline $\begin{array}{l}\text { Lymphocyte } \\
\text { transformation }\end{array}$ & $4.13 \pm 0.23^{\mathrm{a}}$ & $3.89 \pm 0.09^{\mathrm{a}}$ & $3.26 \pm 0.10^{\mathrm{b}}$ & $3.96 \pm 0.10^{\mathrm{a}}$ \\
\hline $\mathrm{IL}-1 \beta(\mathrm{Pg} / \mathrm{ml})$ & $4.37 \pm 0.32^{\mathrm{a}}$ & $4.56 \pm 0.06^{\mathrm{a}}$ & $7.33 \pm 0.32^{\mathrm{b}}$ & $5.03 \pm 0.55^{\mathrm{a}}$ \\
\hline $\mathrm{IL}-12(\mathrm{Pg} / \mathrm{ml})$ & $61.46 \pm 1.11^{\mathrm{b}}$ & $59.07 \pm 0.64^{\mathrm{b}}$ & $177 \pm 2.63^{\mathrm{a}}$ & $114 \pm 3.85^{\mathrm{c}}$ \\
\hline $\mathrm{TAC}(\mathrm{mM} / \mathrm{L})$ & $1.42 \pm 0.0051^{\mathrm{a}}$ & $1.45 \pm 0.0020^{\mathrm{b}}$ & $0.96 \pm 0.0037^{\mathrm{c}}$ & $1.17 \pm 0.0083^{\mathrm{d}}$ \\
\hline $\mathrm{MDA}(\mu \mathrm{mol} / \mathrm{L})$ & $0.64 \pm 0.04^{\mathrm{a}}$ & $0.59 \pm 0.06^{\mathrm{b}}$ & $1.75 \pm 0.12^{\mathrm{a}}$ & $0.89 \pm 0.05^{\mathrm{a}}$ \\
\hline
\end{tabular}

Different superscripts between columns are considered significant at $p<0.05$.

medulla. The BPA group showed marked multifocal lymphocytic depletion. LYC+BPA co-treated group, had a normal cortex and medulla with mild focal lymphocytic depletion (Fig. 3).

\section{Comet assay}

DNA tail as well as \% of damage, in comet results, showed a significant $(p<0.05)$ increase in BPA group. Giving LYC with BPA to experimental rats significantly $(p<0.05)$ ameliorated the ele vated comet tail to a comparable level of control group (Table 3 ).

\section{Discussion}

Current study investigated the adverse effect of BPA on hematological and immunological parameters in Albino rats with testing the protective role of LYC.
The treatment of rats with BPA resulted in significantly increased FCR than control and other groups. However, body weight gain was non-significantly changed. These results were parallel to Ahmed et al. (20). while opposite to those obtained by AbdelRahman et al. (10) who demonstrated a significant decline in the body weight gain in BPA treated female rats. On the other side Rubin et al. (21) demonstrated significant promotion in the weights of female rats' that were prenatally exposed to BPA. This contradictory may be due to sex variation (male may be differed from female) also the difference in doses. The FCR exhibited significant increase in BPA treated rats that may attributed to increase in feed consumption of such group without 
converting to weight gain properly. This may assume to the encountering of BPA to several homeostatic mechanisms inside the body, from which brain neuronal appetite loops (22). The administration of LYC with BPA showed improvement in FCR that denoted the ability of LYC to repair BPA lesion at this point. Hematological findings exhibited non-significant alterations among tested groups. These results concord with Chung et al. (23). However, results were partially agreed with Ahmed et al. (20) who found that BPA ( $150 \mathrm{mg} / \mathrm{kg}$ ) for 70 days reduced RBCs number, PCV and Hb concentration. They found non-significant alterations in MCHC and MCV as well as TLC and DLC. Moreover, Ulutaş et al. (24) mentioned that BPA has no influence on both TLC and DLC. The absence of influence of BPA on hematological picture especially RBCs may be attributed for the shorter duration of exposure and dose that were not enough to affect bone morrow.

Table 3: Effect of BPA and LYC on Comet assay

\begin{tabular}{l|cccc}
\hline Comet assay & Control & LYC & BPA & BPA+LYC \\
\hline \% damage & $15.43^{ \pm 0.18^{\mathrm{b}}}$ & $14.23 \pm 1.13^{\mathrm{b}}$ & $20.67 \pm 1.45^{\mathrm{a}}$ & $17.95^{ \pm 2.34^{\mathrm{b}}}$ \\
\hline DNA tail & $13.49 \pm 0.91^{\mathrm{a}}$ & $14.67 \pm 1.17^{\mathrm{a}}$ & $22.14 \pm 1.28^{\mathrm{b}}$ & $15.39 \pm 2.22^{\mathrm{a}}$ \\
\hline Tail Length & $3.48 \pm 0.82^{\mathrm{a}}$ & $3.49 \pm 0.70^{\mathrm{a}}$ & $5.78 \pm 0.48^{\mathrm{a}}$ & $3.83^{ \pm 0.30^{\mathrm{a}}}$ \\
\hline Tail moment & $0.62 \pm 0.18^{\mathrm{a}}$ & $1.25 \pm 0.27^{\mathrm{a}}$ & $1.17 \pm 0.19^{\mathrm{a}}$ & $0.75 \pm 0.17^{\mathrm{a}}$ \\
\hline
\end{tabular}

Different superscripts between columns are considered significant at $p<0.05$.

Mitogen proliferation assay of lymphocytes was declined significantly after BPA administration than control while BPA and LYC co-administrated reversed this decline. This result was in accordance to Sakazaki et al. ${ }^{(25)}$ who worked on mice. The inferior effect of BPA on blastogenesis of T- cells may be attributed to its capability to generate ROS as well as lipid peroxidation (26) which are observed by decline in serum TAC and MDA levels.

Whereas, oxidative stress can alter cellular proteins as well as lipid causing increase in cellular membrane integrity and electricity ${ }^{(27)}$ thus disrupting cellular function and blastogenesis ability in lymphocytes as an essential step for adaptive immune response. Another evidence supported these results is the elevated level of comet tail and \% of damage in BPA treated group comet results where ROS destructed DNA causing its damage ${ }^{(28,29)}$. The ac- tual mechanism of BPA in inducing oxidative stress and lipid peroxidation is controversial however, literatures supported that it is due to quinones, active metabolites of BPA, that are ROS causing DNA damage (29). These previous effects could be possible cause for the observed thymic and splenic lymphoid depletion observed in the histopathological results. The adverse effects of BPA were ameliorating by co-administration of LYC that manifested by reduced MDA, comet tail and \% damage beside increased lymphocyte transformation and TAC. LYC is considered a potent antioxidant that repaired BPA induced deteriorations and its adverse consequences. Even administration of LYC alone produced significant increase in TAC and reduced MDA than control. This augment the idea that BPA could induce a pro-inflammatory state due to induction of lipid peroxidation and ROS. 


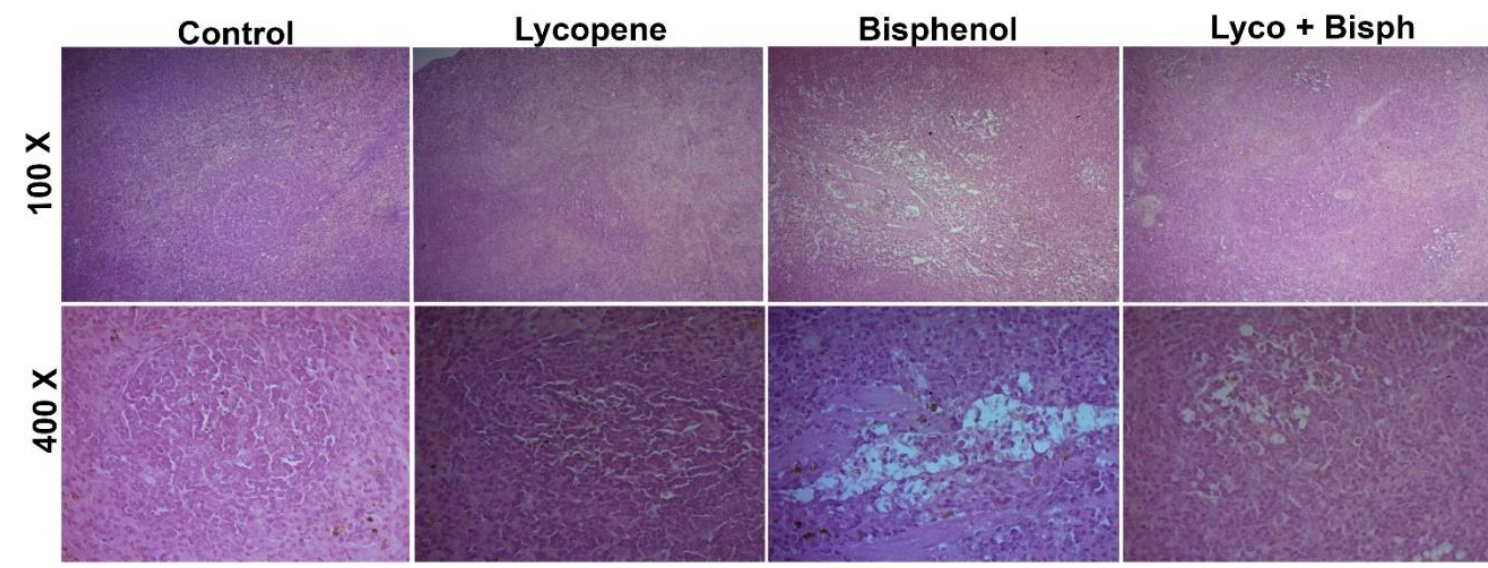

Fig. 2: Microscopic photos showing the histopathological changes of spleen in all study groups. Control and lycopene groups showing normal histological structure. BPA group show marked multifocal depletion of white pulp. Lyco+BPA group show mild depletion of lymphoid follicle. H\&E stain.

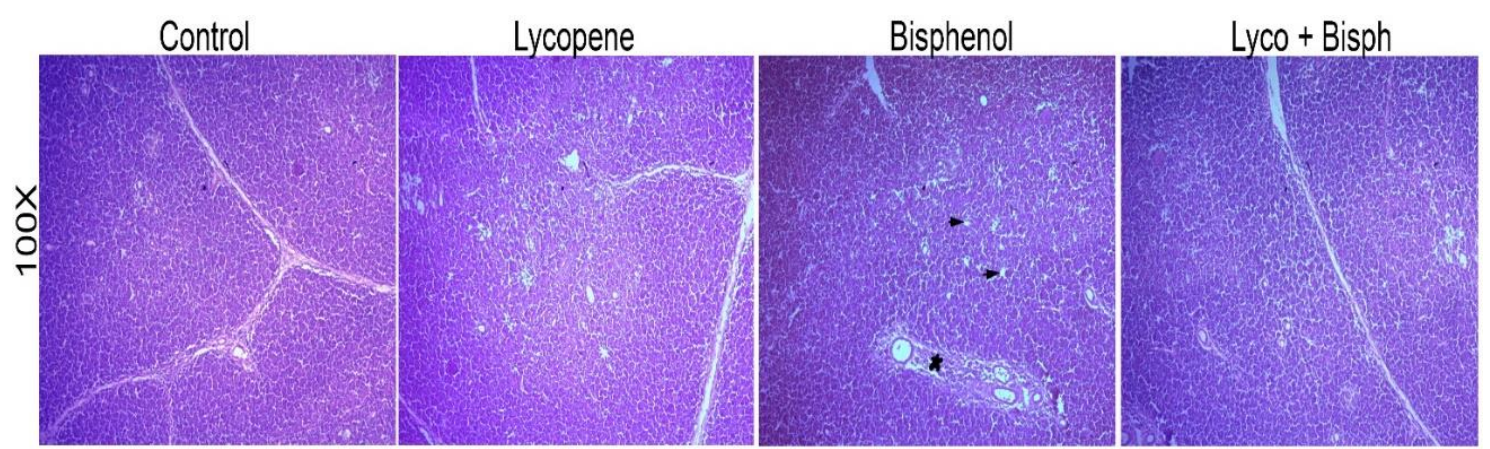

Fig. 3. Microscopic photos showing the histopathological changes of Thymus in all study groups. Thymus showing normal histological structure of both control and lycopene group. PBA group showing multifocal necrotic lymphocytes in cortex and medulla. Lyco+BPA group showing normal thymus. H\&E stain X100.

Oxidative stress promotes nuclear factor-kappa B stimulating various pro-inflammatory cytokines. The promotion of IL-1 $\beta$ indicated that BPA could induce a state of pro-inflammation (31). Induction of IL-12 by BPA is closely associated with $T$-helper 1 cells activation and autoimmunity (32). This adverse effect of BPA could be reduced via administration of LYC, a potential antioxidant that is manifested by ameliorated pro-inflammation and auto- inflammatory $\mathrm{IL}-1 \beta$ and $\mathrm{IL}-12$, respectively. BPA induced immunological perturbations that are represented in suppressed lymphocytes transformation and increased their DNA fragmentation as well as increased IL-1 $\beta$ and IL-12. These perturbations seemed to be induced by ROS and lipid peroxidation inducing effect of BPA. LYC administration ameliorated oxidative stress and lipid peroxidation induced immunological disorders.

\section{References}

1. Karnam SS, C Ghosh R, Mondal S. Effect of bisphenol -a on immune system of wistar rats2016. 47-52 p.

2. Rubin BS. Bisphenol A: an endocrine disruptor with widespread exposure and multiple effects. J Steroid Biochem Mol Biol. 2011;127(1-2):2734. 
3. Sompayrac LM. How the immune system works. WileyBlackwell.2019.

4. Mondal S, Ghosh RC, Mate MS, Karmakar DB. Effects of acetamiprid on immune system in female wistar rats. Proceedings of the Zoological Society. 2009;62(2):109-17.

5. Kuo $\mathrm{CH}$, Yang SN, Kuo PL , Hung $\mathrm{CH}$. Immunomodulatory effects of environmental endocrine disrupting chemicals. The Kaohsiung journal of medical sciences. 2012;28(7 Suppl):S37-42.

6. Gassman NR. Induction of oxidative stress by bisphenol A and its pleiotropic effects. Environmental and molecular mutagenesis. 2017;58(2):60-71.

7. Qiu W, Chen J, Li Y, Chen Z, Jiang $L$, Yang $M$, et al. Oxidative stress and immune disturbance after long-term exposure to bisphenol $A$ in juvenile common carp (Cyprinus carpio). Ecotoxicology and Environmental Safety. 2016;130:93-102.

8. Amirghofran Z. Herbal medicines for immunosuppression. Iranian journal of allergy, asthma, and immunology. 2012;11(2):111-9.

9. Rencuzogullari N, Erdogan S. Oral Administration of Lycopene Reverses Cadmium-suppressed Body Weight Loss and Lipid Peroxidation in Rats. Biological Trace Element Research. 2007;118(2):175-83.

10. Abdel-Rahman HG, Abdelrazek HMA, Zeidan DW, Mohamed RM, Abdelazim AM. Lycopene: Hepatoprotective and Antioxidant Effects toward Bisphenol AInduced Toxicity in Female Wistar Rats. Oxidative medicine and cellular longevity. 2018;2018:5167524.-

11. Jiang $W$, Guo $M-H$, Hai $X$. Hepatoprotective and antioxidant effects of lycopene on non- alcoholic fatty liver disease in rat. World Journal of Gastroenterology. 2016.8-10180:(46)22;

12. Palabiyik SS, Erkekoglu P, Zeybek ND, Kizilgun M, Baydar DE, Sahin $G$, et al. Protective effect of lycopene against ochratoxin A induced renal oxidative stress and apoptosis in rats. Experimental and Toxicologic Pathology. 2013;65(6):85.61-3

13. Chand JN. Shalm's Veterinary Hematology. Lea \& Febiger, Philadelphia.1986.

14. Boyum A. Isolation of mononuclear cells and granulocytes from human blood. Isolation of monuclear cells by one centrifugation, and of granulocytes by combining centrifugation and sedimentation at 1 g. Scand J Clin Lab Invest Suppl. 1968;97:77.

15. Burrells C, Wells PW. In vitro stimulation of ovine lymphocytes by various mitogens. Research in Veterinary Science. 1977;23(1):846.

16. Abdelrazek HMA, Yusuf MS, Ismail SA, Elgawish RA. Effect of probiotic strains mixture administration on serum interleukins concentration, lymphocyte proliferation and DNA damage in rams. Journal of Animal and Feed Sciences. 2015;24(4):3027.

17. Mihara $M$, Uchiyama $M$. Determination of malonaldehyde precursor in tissues by thiobarbituric acid test. Anal Biochem. 1978;86(1):271-8.

18. Drury R, Wallington E. Preparation and fixation of tissues. Carleton's histological technique. 1980;5:4154.

19. Kizilian N, Wilkins R, Reinhardt $P$, Ferrarotto C, McLean J, McNamee J. Silver-stained comet assay for 
detection of apoptosis. Biotechniques. 1999;27(5):926-30.

20. Ahmed W, Moselhy W, Nabil T. Bisphenol A toxicity in adult male rats: hematological, biochemical and histopathological approach. Global veterinaria. 2015;14(2):22838.

21. Rubin BS, Murray MK, Damassa DA, King JC, Soto AM. Perinatal exposure to low doses of bisphenol A affects body weight, patterns of estrous cyclicity, and plasma LH levels. Environmental health perspectives. 2001;109(7):6.80-75

22. Somm E, Schwitzgebel VM, Toulotte A, Cederroth CR, Combescure $C$, Nef $S$, et al. Perinatal exposure to bisphenol a alters early adipogenesis in the rat. Environmental health perspectives. 2009;117(10):154955.

23. Chung YH, Han JH, Lee S-B, Lee Y$\mathrm{H}$. Inhalation toxicity of bisphenol A and its effect on estrous cycle, spatial learning, and memory in rats upon whole-body exposure. Toxicological research. 2017;33(2):165.

24. Ulutaş OK, Yıldız N, Durmaz E, Ahbab MA, Barlas N, Çok I. An in vivo assessment of the genotoxic potential of bisphenol A and 4tert-octylphenol in rats. Archives of toxicology. 2011;85(8):995-1001.

25. Sakazaki H, Ueno H, Nakamuro K. Estrogen receptor $\alpha$ in mouse splenic lymphocytes: possible involvement in immunity. Toxicology letters. 2002;133(23):221-9.

26. Tiwari D, Vanage G. Bisphenol A induces oxidative stress in bone marrow cells, lymphocytes, and reproductive organs of Holtzman rats. International journal of toxicology. 2017;36(2):142-52.

27. Halliwell B, Gutteridge JM .Free radicals in biology and medicine:
Oxford University Press, USA; 2015.

28. Abdelrazek HM, Elgawish RA, Ahmed EA, Bahr HI. In vitro and in vivo effects of Tribulus terrestris on some immunological parameters, lymphocyte proliferation, and DNA integrity in sheep. Small Ruminant Research. 2018;169:67-73.

29. Cavalieri EL, Rogan EG. Is bisphenol $A$ a weak carcinogen like the natural estrogens and diethylstilbestrol? IUBMB life. 2010;62(10):746-51.

30. Guo H, Liu T, Uemura Y, Jiao S-C, Wang D, Lin Z, et al .Bisphenol A in combination with TNF- selectively induces Th2 cell-promoting dendritic cells in vitro with an estrogen-like activity2010. 227-34 p.

31. Elswefy SES, Abdallah FR, Atteia $\mathrm{HH}$, Wahba AS, Hasan RA. Inflammation, oxidative stress and apoptosis cascade implications in bisphenol Ainduced liver fibrosis in male rats. International journal of experimental pathology. 2016;97(5):369-79.

32. Hsieh C, Macatonia S, Tripp C, Wolf S, O'Garra A, Murphy K. Development of TH1 CD4+ T cells through IL-12 produced by Listeriainduced macrophages. Science. 1993;260(5107):547-9. 